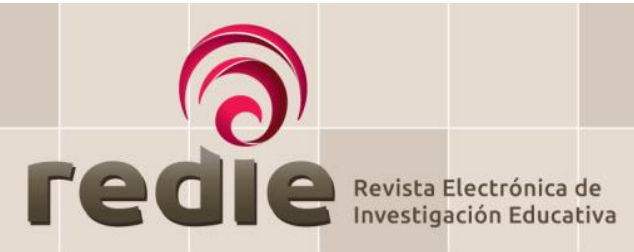

\title{
Percepción del impacto de la evaluación formativa en las competencias profesionales durante la formación inicial del profesorado
}

\section{Perception of Impact of Formative Assessment on Professional Competences During Initial Teacher Training}

Laura Cañadas (1) https://orcid.org/0000-0003-4179-9018

María Luisa Santos Pastor (1) https://orcid.org/0000-0002-4985-0810

Patricia Ruiz Bravo (2) https://orcid.org/0000-0002-4379-4790

(1) Universidad Autónoma de Madrid

(2) Universidad Francisco de Vitoria

(Recibido: 15 de abril de 2019; Aceptado para su publicación: 16 de octubre de 2019)

Cómo citar: Cañadas, L., Santos, M. L. y Ruiz, P. (2021). Percepción del impacto de la evaluación formativa en las competencias profesionales durante la formación inicial del profesorado. Revista Electrónica de Investigación Educativa, 23, e07, 1-13. https://doi.org/10.24320/redie.2021.23.e07.2982

\section{Resumen}

Esta investigación busca evaluar la percepción del impacto que tiene la implementación de experiencias innovadoras de evaluación formativa en el desarrollo de las competencias, así como valorar si existen diferencias en el desarrollo de éstas entre un curso académico y otro. Se contó con 40 experiencias de evaluación formativa en la etapa universitaria. Se empleó un cuestionario con tres escalas de competencias previamente validado que era cumplimentado por el alumnado antes y después de la experiencia de evaluación formativa. De las 45 competencias estudiadas sólo 10 no presentan diferencias significativas entre la valoración final y la inicial; además, no se registran diferencias significativas en la percepción de desarrollo de las competencias estudiadas entre un curso académico y otro.

Palabras clave: evaluación formativa, enseñanza superior, formación inicial

\begin{abstract}
This study aims to assess the perception of the impact of innovative formative assessment experiences on competence development, and determine whether differences exist in competence development from one academic year to the next. A total of 40 university-level formative assessment experiences were taken into account, and a pre-validated questionnaire including three competence scales was completed by students before and after the formative assessment experience. Only 10 of the 45 competences studied exhibited no significant differences between the initial and final evaluation and no significant differences were recorded in perceived competence development between one academic year and the next.
\end{abstract}




\section{Introducción}

La formación inicial del profesorado debe contribuir al desarrollo, en la mayor medida posible, de aquellas competencias que el alumnado necesitará poner en práctica en su futura labor docente, tanto en el caso de los docentes de Educación Primaria como en los de Educación Secundaria (Cardona-Rodríguez et al., 2016; Rodríguez-Gómez et al., 2018). Para ello, la formación inicial debe tener una estructura que permita que a lo largo de los cursos se trabajen las competencias que el alumnado deberá utilizar en su futuro profesional. Como en cualquier titulación deberán desarrollarse las competencias genéricas, aquellas comunes a todas las titulaciones y que se demandan cada vez más en todos los contextos laborales (González y Wagenaar, 2003); y las competencias específicas, que son aquellas propias de una disciplina o de un espacio profesional concreto (Pulido, 2008). Además, en el caso de la formación del profesorado, también será necesario desarrollar competencias docentes genéricas, aquellas que cualquier docente de Primaria o Secundaria debe dominar. Entre estas últimas competencias se encuentran las de participar en la gestión del centro, informar a las familias, gestionar la progresión de los aprendizajes, atender a la diversidad o enfrentarse a los dilemas éticos de la profesión (Marina et al., 2015; Perrenoud, 2004).

Con este propósito, los docentes universitarios deberán emplear estrategias metodológicas y evaluativas que contribuyan a que estos aprendizajes se desarrollen en el mayor grado posible (Hamodi et al., 2017). Uno de los elementos que ha mostrado ser más útil para el aprendizaje de los futuros docentes durante la formación inicial es el empleo de una evaluación formativa (Cañadas, 2018) -elemento educativo por el que se mejoran los procesos de enseñanza y aprendizaje que suceden dentro del aula, para que al finalizar el proceso se consigan los objetivos propuestos (López-Pastor y Sicilia-Camacho, 2017). Desde este punto de vista, la evaluación no puede centrarse exclusivamente en aprendizajes teóricos-memorísticos, sino en habilidades, actitudes, valores, etc., que el alumnado desarrolla en el transcurso del proceso de aprendizaje; por ello, la evaluación formativa se convierte en un elemento clave para la adquisición de las competencias.

La investigación ha mostrado que las competencias clave, como el dominio del proceso de enseñanza, el uso de las TIC y la capacidad organizativa o aprender a aprender se relacionan con la utilización de la evaluación formativa (Romero-Martín et al., 2017). La implementación de procesos de autoevaluación (Biggs, 2005) y evaluación entre iguales (Boud y Falchikov, 2007; Falchikov, 2005) mejoran de manera considerable el aprendizaje de contenidos, desarrollando en los estudiantes procesos metacognitivos y aprendizajes significativos aplicables tanto en su vida académica como en el ámbito profesional. Asimismo, la utilización de instrumentos de evaluación alejados de la concepción tradicional del examen se relaciona positivamente con el desarrollo de competencias genéricas y específicas en la formación inicial de docentes de Educación Física (Cañadas et al., 2018a); y un feedback adecuado se convierte en elemento relevante рага mejorar el aprendizaje del alumnado (Villardón, 2006). Los estudios de Garello y Rinaudo (2013) y Panadero et al. (2018) muestran que la información que recibe el alumnado (de otros o de él mismo) contribuye a mejorar sus aprendizajes.

Las investigaciones relacionadas con el desarrollo de las competencias de los docentes durante esta etapa se han centrado en conocer el grado de desarrollo de las mismas en los últimos cursos o al finalizar las titulaciones, la mayoría dirigidas a la formación de profesorado de Educación Física (EF) (Boned et al., 2006; Campos et al., 2011; Cañadas et al., 2019; Gallardo, 2006; del Valle et al., 2015; Díaz, 2009; Díaz y Aguado, 2012; Kovac et al., 2008; López-Varas, 2015; Martín y de Juanas, 2009; Pazo y Tejada, 2012; RodríguezGómez et al., 2018; Romero, 2009); al respecto, Romero (2009), Pazo y Tejada (2012) y Campos et al. (2011) se enfocan en valorar las competencias más y menos desarrolladas en la titulación de Magisterio de EF (con muestras reducidas, $n<350$ ). Por otra parte, las investigaciones de Boned et al. (2006) y López-Varas (2015) se centran en la percepción de desarrollo de competencias genéricas y específicas de estudiantes de Ciencias de la Actividad Física y el Deporte (CCAFyD). Así, el estudio de Cañadas et al. (2019) cuenta con participantes de ambas titulaciones, y valora la percepción del grado de desarrollo de competencias relacionadas con el conocimiento del contenido y el conocimiento didáctico del contenido en egresados, profesorado y alumnado universitario. En otra dimensión, Kovac et al. (2008) y del Valle et al. (2015) analizan qué tan competentes se sienten los docentes de Primaria y Secundaria en ejercicio de EF en diferentes aspectos relacionados con su tarea docente. 
Sin embargo, los estudios mencionados hasta hora tienen un carácter meramente descriptivo; ninguno de ellos valora cómo la implementación de procesos de evaluación formativa ha contribuido al desarrollo de las competencias profesionales. Sólo Hortigüela et al. (2018) valoran el impacto de procesos de evaluación formativa en las competencias genéricas, encontrando que su utilización contribuye al desarrollo de 4 de los 5 tipos de competencias genéricas estudiadas. Por ello es necesario profundizar y valorar cómo la evaluación formativa puede facilitar el desarrollo de las competencias profesionales durante la formación inicial. El objetivo de esta investigación es valorar la percepción del alumnado sobre el impacto que tiene la implementación de experiencias innovadoras de evaluación formativa en el desarrollo de las competencias genéricas, docentes genéricas y docentes específicas; además, se analizará si existen diferencias en la percepción del grado de desarrollo de competencias entre un curso académico y otro.

\section{Método}

Para esta investigación se empleó un muestreo incidental no probabilístico. Se contó con un total de 40 experiencias innovadoras de evaluación formativa desarrolladas por 29 docentes universitarios. Todos ellos habían realizado anteriormente experiencias de evaluación formativa y pertenecían a la Red de Evaluación Formativa y Compartida en Educación. ${ }^{1}$ En estas experiencias participó un total de 1,959 estudiantes de 15 universidades españolas, en las titulaciones de Maestro en Educación Primaria, Ciencias de la Actividad Física (asignaturas relacionadas con la didáctica y enseñanza de la Educación Física), y un tercer grupo en el que se incluyeron las titulaciones de Maestro en Educación Infantil y Educación Social (materias relacionadas con la pedagogía). En la Tabla 1 se recoge la información descriptiva referida al sexo de participantes, su experiencia docente, las titulaciones en las que se llevaron a cabo las experiencias de evaluación formativa y las universidades donde se desarrollaron; mientras que en la tabla 2 se recogen las asignaturas en las que se implementaron las experiencias de evaluación formativa.

Tabla 1. Datos descriptivos

\begin{tabular}{lcc}
\hline VARIABLE & $\%$ & $\mathrm{~N}$ \\
\hline Sexo & & \\
\hline Hombre & 40 & 16 \\
Mujer & 60 & 24 \\
\hline Experiencia docente & 42.5 & 17 \\
\hline$\leq 10$ años & 30 & 12 \\
Entre 10 y 20 años & 27.5 & 11 \\
$\geq 20$ años & & \\
\hline Titulaciones de procedencia & 40 & 16 \\
Maestro en Educación Primaria & 27.5 & 11 \\
Ciencias de la Actividad Física y el Deporte & 32.5 & 13 \\
Otras & & \\
\hline Universidad & 2.5 & 1 \\
\hline La Laguna & 25 & 10 \\
Universidad de Valladolid & 7.5 & 3 \\
Universidad Autónoma de Barcelona & 7.5 & 3 \\
Universidad Autónoma de Madrid & 2.5 & 1 \\
Universidad de León & 7.5 & 3 \\
Universidad de Burgos & 15 & 6 \\
Universidad de Zaragoza & 5 & 2 \\
Universidad de Alcalá & 2.5 & 1 \\
Universidad de Murcia & 2.5 & 1 \\
Universidad de Castilla La Mancha & 10 & 4 \\
Universidad de Valencia & 5 & 2 \\
Universidad de Cantabria & 2.5 & 1 \\
Universidad de Córdoba & 2.5 & 1 \\
Universidad Católica de Murcia (UCAM) & 2.5 & 1 \\
Universidad de Vigo & & \\
\hline & & \\
\hline
\end{tabular}

${ }^{1}$ https://redevaluacionformativa.wordpress.com 
Tabla 2. Asignaturas en las que se implementaron las experiencias de evaluación formativa

\begin{tabular}{|c|c|c|}
\hline Titulación & Asignatura & $\mathrm{N}$ veces \\
\hline \multirow{11}{*}{$\begin{array}{l}\text { Maestro en } \\
\text { Educación Primaria }\end{array}$} & Aprendizaje y desarrollo motor & 1 \\
\hline & Ciencia, tecnología y sociedad & 1 \\
\hline & Didáctica de la Educación Física (o Educación Física y su didáctica) & 6 \\
\hline & Didáctica de la lengua oral y escrita & 1 \\
\hline & Educación Física y salud & 1 \\
\hline & Formas de expresión musical & 1 \\
\hline & Fundamentos y didáctica de la educación corporal & 1 \\
\hline & Métodos de Investigación e Innovación en Educación & 1 \\
\hline & Prácticum I & 1 \\
\hline & Prácticum III (Especialidad Educación Física) & 1 \\
\hline & Trabajo Fin de Grado & 1 \\
\hline \multirow{9}{*}{$\begin{array}{l}\text { Ciencias de la } \\
\text { Actividad Física y el } \\
\text { Deporte }\end{array}$} & Actividades físico-deportivas en el medio natural & 1 \\
\hline & Actividades corporales de expresión & 2 \\
\hline & Diseño y análisis de la enseñanza de la actividad física & 1 \\
\hline & Diseño, intervención y evaluación de la actividad física y deporte & 1 \\
\hline & $\begin{array}{l}\text { Diseño, organización y desarrollo de actividades para la enseñanza de la } \\
\text { Educación Física }\end{array}$ & 1 \\
\hline & Educación Física curricular & 1 \\
\hline & Enseñanza de la actividad física y el deporte II & 2 \\
\hline & Iniciación deportiva & 1 \\
\hline & Procesos de enseñanza-aprendizaje de la actividad física y el deporte & 1 \\
\hline \multirow{8}{*}{ Otros } & Didáctica de la Educación Corporal en Educación Infantil & 2 \\
\hline & Didáctica de la Educación Física en Educación infantil & 3 \\
\hline & Didáctica del medio natural social y cultural & 1 \\
\hline & Diseño y evaluación en la enseñanza de la actividad física y del deporte & 1 \\
\hline & $\begin{array}{l}\text { Diseño, organización y desarrollo de actividades para la enseñanza de la } \\
\text { Educación Física }\end{array}$ & 1 \\
\hline & Educación para la paz y la igualdad & 3 \\
\hline & Expresión y comunicación corporal en Educación Infantil & 1 \\
\hline & Modelos de negocio y estrategias web & 1 \\
\hline Total & & 40 \\
\hline
\end{tabular}

Para la recogida de información se utilizó un cuestionario con tres escalas previamente validadas (Salcines et al., 2018): una escala de competencias genéricas $\left(C_{g}\right)$, una de competencias docentes genéricas ( $\left.C_{d g}\right)$ y una de competencias docentes específicas ( $C_{\text {de) }}$. La escala $C_{g}$ está compuesta por 14 ítems que recogen las competencias genéricas que se deben desarrollar en las diferentes titulaciones universitarias (entre otras, conocer una lengua extranjera, utilizar las TIC, desarrollar habilidades inter e intra personales, trabajar en equipo, etc.); la de $C_{d g}$ está formada por 17 ítems que aglutinan aspectos como conocer las características de los centros educativos, diseñar y animar situaciones de aprendizaje, diseñar y poner en práctica estrategias de atención a la diversidad, etc.; y la escala de $C_{\text {de }}$ está formada por 14 ítems que recogen aspectos relacionados con la programación, intervención y evaluación de procesos de enseñanza y aprendizaje, diferentes contenidos motrices, deportivos y de salud, etc. Todas las preguntas se contestaron con una escala Likert de 4 puntos, donde 1 (nada); 2 (poco); 3 (bastante); 4 (mucho).

Se realizó estudio de corte transversal; el cuestionario se entregaba físicamente al alumnado de las diferentes asignaturas donde se iban a llevar a cabo las experiencias de evaluación formativa. Antes de comenzar la asignatura concreta en la que se iba a realizar la experiencia de evaluación formativa se les pasó el cuestionario con la pregunta: "Valora en qué grado crees que en estos momentos tienes la capacidad de...". Este mismo cuestionario se entregó también al final de la asignatura con la pregunta: "Valora el grado en que crees que esta asignatura te ha ayudado a desarrollar la capacidad de...". Los datos se recogieron en los cursos 2015-2016 y 2016-2017; una vez recogidos los datos se calculó el valor medio para cada ítem, siendo ese el valor de la competencia en la experiencia de evaluación formativa. Al alumnado se le informó sobre la finalidad de la investigación y se garantizó la confidencialidad de los datos. 
Las experiencias de evaluación formativa desarrolladas por el profesorado universitario se recogían en un informe final de manera que se asegurase que todas cumplían los requisitos para ser considerados experiencias de evaluación formativa. En todas ellas el objetivo era la mejora del proceso de enseñanza y aprendizaje y la evaluación se realizaba de forma continua a lo largo de todo el proceso, y no de forma puntual al final de éste; se daba feedback al alumnado a lo largo de las diferentes tareas realizadas en la asignatura; en la mayoría de las experiencias se presentaban instrumentos de evaluación innovadores para la evaluación de los aprendizajes del alumnado (portafolios, rúbricas, etc.); y, en algunas de estas experiencias el alumnado participaba en su evaluación (autoevaluación), en la de sus compañeros (coevaluación) e incluso en algunas experiencias la evaluación era compartida con el docente. Cada uno de los docentes que implementaba la experiencia decidía qué aspecto innovador introduciría en el curso académico en su materia, siempre y cuando la experiencia implementada cumpliese con las características de una verdadera evaluación formativa. Por ejemplo, ésta podría estar justificada por la utilización de un nuevo instrumento de evaluación, el empleo de alguna forma de evaluación participativa, etc.

Para la realización de los análisis se utilizó el paquete estadístico SPSS v.21. Se presenta la media y la desviación típica de cada una de las competencias tanto en la valoración inicial como en la final. Se comprobó la normalidad de la muestra con la prueba de Kolmogorov-Smirnov, presentando todos los ítems valores mayores de 0.05 , por tanto, una distribución normal. Los datos iniciales y finales se analizaron utilizando diferencias de medias inter-sujetos. Para valorar si se han desarrollado las competencias tras la implementación de la experiencia de evaluación formativa se utilizó la t de Student para muestras relacionadas. Se calculó la diferencia existente entre el desarrollo de competencias inicial y final para cada una de las variables ( $\left.C_{91 \text { dif }}=C_{g 1 \text { final }}-C_{g 1 \text { inicial }}\right)$. Posteriormente se calculó un valor medio final para cada tipo de competencias $\left(\Sigma C_{g 1 \text { dif }}+C_{g 2 d i f}+\ldots C_{g n d i f}\right) / n$ y se valoró si existían diferencias entre el desarrollo de competencias entre un curso académico y otro. Para ello se empleó la $t$ de Student para muestras independientes. El nivel de significación de todos los análisis se estableció en $p<0.05$.

\section{Resultados}

En la Tabla 3 se recoge la diferencia de percepción del alumnado entre la valoración final e inicial del grado de desarrollo de competencias genéricas. Sólo hay dos competencias donde no se valora que haya habido una mejora: comunicarse de forma oral y escrita en lengua nativa $(\rho=0.235)$ y utilizar las TIC en el ámbito de estudio ( $p=0.358)$. Aquellas donde se ha producido un mayor incremento son: razonar de forma crítica y reflexiva $(d=-1.380 ; p=0.000)$; desarrollar la creatividad $(d=-1.340 ; p=0.000)$; trabajar en equipo $(d=$ $1.074 ; p=0.000) ; y$ adaptarse a situaciones nuevas $(d=-1.060 ; p=0.000)$. 
Tabla 3. Diferencia entre el pre-test y el post-test en la percepción de desarrollo de las competencias genéricas

\begin{tabular}{|c|c|c|c|c|c|}
\hline & Inicial & Final & $t$ & $d$ & $p$ \\
\hline $\mathrm{n}$ & 40 & 40 & & & \\
\hline 1.1 Analizar y sintetizar & $2.82(0.27)$ & $3.04(0.27)$ & -4.415 & -0.806 & 0.000 \\
\hline 1.2 Organizar y planificar & $2.95(0.37)$ & $3.25(0.34)$ & -4.794 & -0.830 & 0.000 \\
\hline 1.3 Comunicarse de forma oral y escrita en lengua & $3.00(0.50)$ & $3.11(0.36)$ & -1.207 & -0.572 & 0.235 \\
\hline $\begin{array}{l}\text { 1.4 Comunicarse gestual y corporalmente } \\
1.5 \text { Conocer una lengua extranjera }\end{array}$ & $\begin{array}{l}2.84(0.31) \\
2.19(0.44)\end{array}$ & $\begin{array}{l}3.12(0.53) \\
1.92(0.67)\end{array}$ & $\begin{array}{l}-2.705 \\
2.210\end{array}$ & $\begin{array}{c}-0.644 \\
0.477\end{array}$ & $\begin{array}{l}0.010 \\
0.033\end{array}$ \\
\hline $\begin{array}{l}\text { 1.6 Utilizar las Tecnologías de la Información y la } \\
\text { Comunicación en el ámbito de estudio }\end{array}$ & $2.76(0.44)$ & $2.87(0.55)$ & -0.931 & -0.223 & 0.358 \\
\hline $\begin{array}{l}\text { 1.7 Trabajar en equipo } \\
\text { 1.8 Desarrollar habilidades en las relaciones }\end{array}$ & $3.21(0.35)$ & $3.61(0.41)$ & -5.715 & -1.074 & 0.000 \\
\hline $\begin{array}{l}\text { interpersonales (empatía, asertividad, respeto y } \\
\text { escucha) }\end{array}$ & $3.20(0.44)$ & $3.43(0.34)$ & -2.720 & -0.586 & 0.010 \\
\hline $\begin{array}{l}\text { 1.9 Desarrollar habilidades intrapersonales } \\
\text { (autoestima, motivación y confianza en uno mismo) }\end{array}$ & $2.89(0.53)$ & $3.24(0.40)$ & -3.302 & -0.750 & 0.002 \\
\hline 1.10 Razonar de forma crítica y reflexiva & $2.91(0.28)$ & $3.30(0.30)$ & -5.598 & -1.380 & 0.000 \\
\hline 1.11 Desarrollar un compromiso ético & $2.91(0.48)$ & $3.15(0.41)$ & -2.669 & -0.550 & 0.011 \\
\hline 1.12 Desarrollar un aprendizaje autónomo & $2.95(0.41)$ & $3.27(0.35)$ & -3.976 & -0.873 & 0.000 \\
\hline 1.13 Adaptarse a situaciones nuevas & $2.97(0.32)$ & $3.34(0.38)$ & -5.615 & -1.060 & 0.000 \\
\hline 1.14 Desarrollar la creatividad & $2.84(0.31)$ & $3.29(0.36)$ & -6.916 & -1.340 & 0.000 \\
\hline
\end{tabular}

Nota: En negrita diferencias significativas.

En la Tabla 4 se recoge la diferencia entre la valoración final e inicial de la percepción del alumnado del grado de desarrollo de competencias docentes genéricas. Hay cinco competencias que no muestran diferencias significativas entre la valoración final y la inicial, todas ellas relacionadas con el papel del profesorado tanto en implicarse él mismo como a los otros en la vida y gestión del centro, como en implicar a las familias en el aprendizaje de sus hijos. Estas competencias son: implicar al alumnado en la vida del centro $(p=0.216)$; participar en la gestión del centro $(p=0.114)$; informar a las familias sobre la evolución de su hijo ( $p=0.947)$; implicar a las familias en el aprendizaje de su hijo ( $p=0.647)$; e implicar a las familias en la vida del centro $(p=0.786)$. En cuanto a las competencias más desarrolladas, están: elaborar propuestas de cambio de la realidad educativa $(d=-1.837 ; p=0.000)$; diseñar situaciones de aprendizaje $(d$ $=-1.664 ; p=0.000) ;$ y gestionar la progresión de los aprendizajes $(d=-1.507 ; p=0.000)$. 
Tabla 4. Diferencia entre el pre-test y el post-test en la percepción de desarrollo de las competencias docentes genéricas

\begin{tabular}{|c|c|c|c|c|c|}
\hline & Inicial & Final & $t$ & $d$ & $p$ \\
\hline$n$ & 40 & 40 & & & \\
\hline $\begin{array}{l}\text { 2.1 Conocer las características organizativas de los } \\
\text { centros educativos }\end{array}$ & $2.31(0.50)$ & $2.61(0.50)$ & -2.605 & -0.597 & 0.013 \\
\hline $\begin{array}{l}\text { 2.2 Elaborar propuestas de cambio de la realidad } \\
\text { educativa }\end{array}$ & $2.43(0.23)$ & $3.03(0.41)$ & -8.338 & -1.837 & 0.000 \\
\hline 2.3 Diseñar situaciones de aprendizaje & $2.75(0.28)$ & $3.37(0.45)$ & -7.184 & -1.664 & 0.000 \\
\hline 2.4 Animar situaciones de aprendizaje & $2.94(0.22)$ & $3.31(0.41)$ & -6.623 & -1.153 & 0.000 \\
\hline 2.5 Gestionar la progresión de los aprendizajes & $2.55(0.40)$ & $3.09(0.33)$ & -5.436 & -1.507 & 0.000 \\
\hline 2.6 Diseñar estrategias de atención a la diversidad & $2.39(0.54)$ & $2.90(0.50)$ & -4.008 & -0.994 & 0.000 \\
\hline $\begin{array}{l}\text { 2.7 Poner en práctica estrategias de atención a la } \\
\text { diversidad }\end{array}$ & $2.35(0.59)$ & $2.72(0.48)$ & -3.248 & -0.692 & 0.002 \\
\hline 2.8 Implicar al alumnado en su aprendizaje & $2.83(0.40)$ & $3.28(0.35)$ & -5.325 & -1.192 & 0.000 \\
\hline 2.9 Implicar al alumnado en la vida del centro & $2.53(0.44)$ & $2.66(0.54)$ & -1.259 & -0.267 & 0.216 \\
\hline 2.10 Participar en la gestión del centro & $2.14(0.44)$ & $2.28(0.46)$ & -1.620 & -0.334 & 0.114 \\
\hline 2.11 Informar a las familias sobre la evolución de su hijo & $2.35(0.59)$ & $2.34(0.63)$ & 0.067 & 0.015 & 0.947 \\
\hline 2.12 Implicar a las familias en el aprendizaje de su hijo & $2.35(0.56)$ & $2.42(0.67)$ & 0.462 & -0.119 & 0.647 \\
\hline 2.13 Implicar a las familias en la vida del centro & $2.30(0.47)$ & $2.33(0.79)$ & 0.273 & -0.048 & 0.786 \\
\hline $\begin{array}{l}\text { 2.14 Afrontar los deberes y dilemas éticos de la } \\
\text { profesión }\end{array}$ & $2.47(0.53)$ & $2.82(0.33)$ & -3.404 & -0.822 & 0.002 \\
\hline $\begin{array}{l}2.15 \text { Atender al propio desarrollo profesional docente } \\
\text { (autoevaluación, lecturas, cursos, jornadas, congresos, } \\
\text { redes de trabajo) }\end{array}$ & $2.59(0.32)$ & $3.06(0.46)$ & -5.749 & -1.209 & 0.000 \\
\hline $\begin{array}{l}\text { 2.16 Desarrollar procesos de innovación educativa en el } \\
\text { aula }\end{array}$ & $2.56(0.38)$ & $3.11(0.43)$ & -5.921 & -1.377 & 0.000 \\
\hline $\begin{array}{l}\text { 2.17 Poner en marcha procesos de investigación en el } \\
\text { aula }\end{array}$ & $2.33(0.33)$ & $2.85(0.60)$ & -4.620 & -1.093 & 0.000 \\
\hline
\end{tabular}

Nota: En negrita diferencias significativas.

En la Tabla 5 se presenta la diferencia entre la valoración final e inicial de la percepción del alumnado del grado de desarrollo de competencias docentes específicas. Se utilizaron únicamente los datos de aquellas experiencias que habían sido llevadas a cabo en alguna asignatura de las titulaciones relacionadas con la actividad física y el deporte. Hay tres competencias que no presentan diferencias significativas en el grado de desarrollo entre la valoración final y la inicial, esta son: identificar los riesgos que se derivan para la salud de la práctica de actividades físicas inadecuadas ( $p=0.819$ ); diseñar un plan de hábitos de vida saludable (higiene y alimentación) y de práctica de actividad física regular $(p=0.380)$; y saber aplicar los fundamentos (técnicas) de las actividades físicas en el medio natural $(p=0.294)$. Y las competencias que presentan un mayor desarrollo son: saber utilizar diferentes instrumentos de evaluación en el área de Educación Física ( $d$ $=-1.396 ; p=0.000$ ); Diseñar, desarrollar y evaluar los procesos de enseñanza-aprendizaje relativos a la actividad física y el deporte con atención a las Necesidades Específicas de Apoyo Educativo $(d=-1.243 ; p=$ 0.000); y tener capacidad de reflexión sobre el proceso de enseñanza/aprendizaje y las distintas metodologías dentro de las clases de Educación Física $(d=-1.168 ; p=0.000)$. 
Tabla 5. Diferencia entre el pre-test y el post-test en la percepción de desarrollo de las competencias docentes específicas

\begin{tabular}{|c|c|c|c|c|c|}
\hline & Inicial & Final & $t$ & $d$ & $p$ \\
\hline$n$ & 27 & 27 & & & \\
\hline $\begin{array}{l}\text { 3.1 Diseñar, aplicar y analizar intervenciones didácticas } \\
\text { en el área de Educación Física }\end{array}$ & $2.49(0.49)$ & $3.07(0.56)$ & -5.526 & -1.100 & 0.000 \\
\hline $\begin{array}{l}\text { 3.2 Diseñar, desarrollar y evaluar los procesos de } \\
\text { enseñanza-aprendizaje relativos a la actividad física y el } \\
\text { deporte con atención a las Necesidades Específicas de } \\
\text { Apoyo Educativo }\end{array}$ & $2.36(0.39)$ & $2.91(0.51)$ & -6.259 & -1.243 & 0.000 \\
\hline $\begin{array}{l}\text { 3.3 Conocer y promover las diferentes manifestaciones } \\
\text { motrices que forman parte de la cultura tradicional }\end{array}$ & $2.44(0.40)$ & $2.75(0.46)$ & -3.532 & -0.839 & 0.002 \\
\hline $\begin{array}{l}\text { 3.4 Conocer y aplicar los fundamentos biológicos, } \\
\text { fisiológicos, de maduración evolutiva y el desarrollo } \\
\text { psicomotor }\end{array}$ & $2.41(0.30)$ & $2.71(0.44)$ & -2.886 & -0.801 & 0.008 \\
\hline $\begin{array}{l}\text { 3.5 Diseñar, modificar y/o adaptar al contexto } \\
\text { educativo situaciones motrices orientadas al desarrollo } \\
\text { y perfeccionamiento de las habilidades motrices }\end{array}$ & $2.51(0.41)$ & $2.80(0.68)$ & -2.478 & -0.525 & 0.020 \\
\hline $\begin{array}{l}\text { 3.6 Conocer los elementos y fundamentos de la } \\
\text { expresión corporal y la comunicación no verbal y su } \\
\text { valor formativo y cultural }\end{array}$ & $2.55(0.44)$ & $2.88(0.54)$ & -4.201 & -0.670 & 0.000 \\
\hline $\begin{array}{l}\text { 3.7 Saber utilizar el juego como recurso didáctico y } \\
\text { como contenido de enseñanza }\end{array}$ & $2.98(0.40)$ & $3.23(0.45)$ & -2.950 & -0.600 & 0.007 \\
\hline $\begin{array}{l}\text { 3.8 Conocer los fundamentos básicos de la iniciación } \\
\text { deportiva escolar y diseñar tareas específicas para } \\
\text { utilizarlos en el ámbito de la enseñanza }\end{array}$ & $2.61(0.43)$ & $2.98(0.54)$ & -4.357 & -0.770 & 0.000 \\
\hline $\begin{array}{l}3.9 \text { Identificar los riesgos que se derivan para la salud } \\
\text { de la práctica de actividades físicas inadecuadas }\end{array}$ & $2.71(0.31)$ & $2.74(0.42)$ & -0.231 & -0.066 & 0.819 \\
\hline $\begin{array}{l}\text { 3.10 Diseñar un plan de hábitos de vida saludable } \\
\text { (higiene y alimentación) y de práctica de actividad física } \\
\text { regular }\end{array}$ & $2.76(0.34)$ & $2.67(0.44)$ & 0.894 & 0.254 & 0.380 \\
\hline $\begin{array}{l}\text { 3.11 Saber aplicar los fundamentos (técnicas) de las } \\
\text { actividades físicas en el medio natural }\end{array}$ & $2.57(0.40)$ & $2.68(0.53)$ & -1.071 & -0.235 & 0.294 \\
\hline $\begin{array}{l}\text { 3.12 Saber utilizar diferentes instrumentos de } \\
\text { evaluación en el área de Educación Física }\end{array}$ & $2.49(0.48)$ & $3.17(0.51)$ & -7.152 & -1.396 & 0.000 \\
\hline $\begin{array}{l}\text { 3.13 Tener capacidad de reflexión sobre el proceso de } \\
\text { enseñanza/aprendizaje y las distintas metodologías } \\
\text { dentro de las clases de Educación Física }\end{array}$ & $2.54(0.52)$ & $3.15(0.54)$ & -6.442 & -1.168 & 0.000 \\
\hline $\begin{array}{l}\text { 3.14 Analizar y comunicar, de manera crítica y } \\
\text { fundamentada, el valor de la actividad física y el } \\
\text { deporte y sus posibilidades de contribuir al desarrollo y } \\
\text { bienestar de las personas }\end{array}$ & $2.76(0.47)$ & $3.14(0.36)$ & -3.879 & -0.922 & 0.001 \\
\hline
\end{tabular}

Nota: En negrita diferencias significativas.

Por último, se valoró si existían diferencias en la percepción del grado de desarrollo de cada grupo de competencias entre cursos académicos. Estos datos se presentan en la Tabla 6. No existen diferencias significativas en ninguno de los grupos de competencias entre un curso académico y otro $(p>0.05)$. Se comprobó si individualmente alguna de las competencias presentaba diferencias significativas entre un curso y otro, y no se encontraron en ninguna. 
Tabla 6. Diferencias entre grupos de competencias en función del curso académico en que se llevó a cabo la experiencia de evaluación formativa

\begin{tabular}{lcccc}
\hline & $2015 / 2016$ & $2016 / 2017$ & $t$ & $p$ \\
\hline $\mathrm{n}$ & 14 & 26 & & \\
\hline Competencias genéricas & $0.35(0.59)$ & $0.19(0.31)$ & 1.109 & 0.279 \\
Competencias docentes genéricas & $0.53(0.71)$ & $0.26(0.44)$ & 1.462 & 0.152 \\
\hline $\mathrm{n}$ & 12 & 15 & & 0.518 \\
\hline Competencias docentes específicas & $0.38(0.46)$ & $0.30(0.34)$ & 0.609 \\
\hline
\end{tabular}

\section{Discusión y conclusión}

Los resultados de esta investigación desvelan que la utilización de la evaluación formativa en la enseñanza universitaria contribuye tanto al desarrollo de las competencias genéricas como al de las docentes genéricas y específicas. De las 45 competencias estudiadas en total, sólo 10 de ellas no presentan diferencias entre la valoración final y la inicial. El resto de las competencias muestran un incremento en la percepción que el alumnado tiene de su adquisición tras la implementación de experiencias innovadoras de evaluación formativa. Además, no se encontraron diferencias significativas en la percepción del desarrollo de las competencias estudiadas entre un curso académico y otro.

Respecto a las competencias genéricas la investigación mostró que tras la implementación de experiencias innovadoras de evaluación formativa se produjo un incremento en casi todas ellas, especialmente las relacionadas con razonar de forma crítica, adaptarse a situaciones novedosas y desarrollar la creatividad. Incluso, como se detecta en otras investigaciones (Gallardo, 2006; López-Varas, 2015), se manifiesta una mejora en aquellas vinculadas con el trabajo colaborativo o en equipo, con las relaciones interpersonales y el compromiso ético. En este sentido, podemos pensar que los procedimientos asociados a la puesta en práctica de la evaluación formativa (co-evaluación, autoevaluación) promueven per se una actitud (auto)crítica y (auto)reflexiva que redunda de manera directa en la adquisición de estas competencias (Martínez-Izaguirre et al., 2018), siempre que haya habido una coherencia y alineación entre las acciones formativas de la evaluación y las competencias a fomentar. Una situación similar se detecta en relación con el desarrollo de competencias docentes genéricas, al observar que se producen importantes repercusiones en algunas de ellas, como: elaborar propuestas de cambio de la realidad educativa; diseñar situaciones de aprendizaje; y gestionar la progresión de los aprendizajes. Este efecto podría atribuirse a los protocolos/acciones que se promueven en las tareas planteadas en los procesos de evaluación formativa, y que son referidos a los ciclos de acción-reflexión y cambio.

Esta teoría también podría ayudarnos a explicar la nula o escasa incidencia que tiene la evaluación formativa en el desarrollo de algunas competencias genéricas. En concreto, coincidiendo con Hortigüela et al. (2018), se detecta que no ha habido una mejora en competencias relacionadas con la comunicación oral y escrita en lengua nativa ni en el uso de las TIC. Estas competencias resultan ser las menos valoradas en aquellos estudios donde se investiga el grado de adquisición de las competencias al finalizar el período formativo (Gallardo, 2006; López-Varas, 2015; Pazo y Tejada, 2012). Parece, por tanto, que la utilización de procesos de evaluación formativa no contribuirá a solventar esa falta de formación y deberán ponerse en marcha otras estrategias para conseguir que al finalizar las titulaciones se hayan desarrollado en mayor medida. De manera similar, se percibe la poca incidencia de la evaluación formativa sobre ciertas competencias docentes genéricas, como las referidas a la gestión del centro e implicar a las familias en el aprendizaje y la vida del centro. Éstas, tal como se ha planteado en otras investigaciones (Cañadas, 2018), son de las competencias que se aprecia se han desarrollado menos al finalizar la formación inicial. Por tanto, como ocurría con algunas competencias genéricas, parece que, al no tratarse de competencias vinculadas específicamente con procesos de evaluación formativa, la escasa incidencia sobre estas competencias docentes genéricas queda justificada. 
Asimismo, se descubre que las competencias docentes específicas obtienen grandes beneficios por la implementación de experiencias innovadoras de evaluación formativa. Incluso se comprueba que las que consiguen mayor valoración son las competencias vinculadas con los contenidos específicos de la disciplina. Sin embargo, algunas investigaciones (Cañadas et al., 2019) han mostrado que se encuentran valoraciones distintas en función de la titulación que hayan cursado los participantes (Magisterio de Educación Física vs. CCAFyD). Habría que valorar si en el caso de la implementación de estas experiencias de evaluación formativa ocurre lo mismo.

Además, dentro de las competencias docentes específicas, la vivencia de experiencias de evaluación formativa también contribuirá al desarrollo de aquellas relacionadas con el contenido pedagógico que implican procesos de diseño e intervención metodológica como diseñar, desarrollar y evaluar los procesos de enseñanza-aprendizaje relativos a la actividad física y el deporte con atención a las Necesidades Específicas de Apoyo Educativo; o que supongan poner en práctica procesos de evaluación en EF, como saber utilizar diferentes instrumentos de evaluación en el área de Educación Física; y procesos de reflexión crítica sobre la práctica de la EF, como tener capacidad de reflexión sobre el proceso de enseñanza/aprendizaje y las distintas metodologías dentro de las clases de Educación Física. Se vuelve a manifestar la importancia de conectar los aprendizajes de formación inicial con las acciones de la práctica profesional (Ní Chróinín y O'Sullivan, 2014). Es decir, adquirir la competencia relacionada con la evaluación formativa implicará hacer evaluación formativa en la Universidad (Hamodi et al., 2017), o lo que es lo mismo, practicar la evaluación formativa durante la formación inicial tendrá consecuencias en el desarrollo de las competencias docentes específicas.

Por otra parte, se observa que no se produce tanta influencia en otras competencias docentes específicas como son la de identificar los riesgos que se derivan para la salud de la práctica de actividades físicas inadecuadas; diseñar un plan de hábitos de vida saludable (higiene y alimentación) y de práctica de actividad física regular; y saber aplicar los fundamentos (técnicas) de las actividades físicas en el medio natural. Es posible que esto se deba al carácter más procedimental que se aplica en estos aprendizajes o a la escasa inclusión de estos contenidos técnicos con los procesos de evaluación formativa que se implantan. A pesar de ello, la información recabada no permite hacer una valoración más profunda para saber en qué grado se han alcanzado estas competencias.

Las principales limitaciones que presenta este estudio se encuentran en el tipo de muestreo empleado, ya que al ser incidental no permite generalizar los resultados. El diseño transversal de esta investigación también representa una limitación. Además, también se valora la percepción del alumnado de adquisición de las competencias, y no el verdadero grado de adquisición de estas. En cuanto a las competencias específicas, solamente se valoran las del alumnado de titulaciones dirigidas a la formación del profesorado de Educación Física, quedando sin estimar las competencias específicas de otras especialidades. Entre las fortalezas que presenta esta investigación está la utilización de datos con una amplia muestra de alumnado procedente de un variado número de universidades, recogidos en dos periodos diferentes. Además, se valoran tanto las competencias genéricas, como las docentes genéricas y las específicas, dando una amplia visión del posible impacto de las experiencias de evaluación formativa.

Consideramos que son necesarios más estudios que ahonden en cómo los procesos de evaluación formativa impactan en el desarrollo de las competencias profesionales en la formación inicial del profesorado. Una futura línea de investigación sería valorar cómo las acciones que conforman los procesos de evaluación (feedback, instrumentos, participación del alumnado, etc.) condicionan o impactan en cada tipo de competencia. Además, sería necesario indagar las limitaciones que percibe el estudiante en la adquisición de competencias durante su formación inicial, cuáles son desatendidas y cómo se podría solventar este olvido.

El estudio realizado muestra que la evaluación formativa parece tener un importante impacto en el desarrollo de los aprendizajes del alumnado durante la formación universitaria. Así, en los procesos de enseñanza y aprendizaje no se puede dejar de lado o descontextualizar los procesos evaluativos. Tan 
importante es el enfoque metodológico elegido para la adquisición de los aprendizajes como la coherencia de éste con la evaluación; o el empleo de una evaluación que fomente la constante mejora del aprendizaje, y no únicamente valorar el grado en que se han adquirido los aprendizajes con el objetivo de asignarles una calificación (Cañadas, 2018; Cañadas et al., 2018b). Como se muestra en esta investigación, se percibe que el empleo de procesos de evaluación formativa contribuye al desarrollo de las competencias (genéricas, docentes genéricas y docentes específicas) en la formación inicial del profesorado.

Dados los resultados de esta investigación parece necesario seguir profundizando en el papel que juega la evaluación formativa y en qué medida influye en el aprendizaje del alumnado, así como en la adquisición de las competencias propias de la titulación. Además, es necesario seguir formando al profesorado universitario en el empleo de formas de evaluación democráticas y participativas, coherentes con una formación basada en competencias, vinculada con las necesidades reales del contexto laboral.

\section{Referencias}

Biggs, J. B. (2005). Calidad del aprendizaje universitario. Narcea.

Boned, C. J., Rodríguez-Romo, G., Mayorga, J. I. y Merino, A. (2006). Competencias profesionales del Licenciado en Ciencias de la Actividad Física y del Deporte. Motricidad, 15, 1-6.

Boud, D. y Falchikov, N. (2007). Rethinking assessment in higher education: learning for the long term. Routledge.

Campos, M. C., Ries, F. y Del Castillo, O. (2011). Análisis de las competencias adquiridas y utilizadas por los egresados maestros en Educación Física. Revista Internacional de Ciencias del Deporte, 7(24), 216-229. https://doi.org/10.5232/ricyde2011.02405

Cañadas, L. (2018). La evaluación formativa en la adquisición de competencias docentes en la formación inicial del profesorado de educación Física [Tesis Doctoral Inédita]. Universidad Autónoma de Madrid.

Cañadas, L., Santos-Pastor, M. L. y Castejón, F. J. (2018a). Desarrollo de competencias docentes en la formación inicial del profesorado de educación física. Relación con los instrumentos de evaluación. Estudios Pedagógicos, 44(2), 111-126. http://dx.doi.ora/10.4067/S0718-07052018000200111

Cañadas, L., Santos-Pastor, M. L. y Castejón, F. J. (2018b). Evaluación en la formación inicial. ¿Avance o retroceso? Bordón. Revista de Pedagogía, 70(4), 9-22. https://doi.org/10.13042/Bordon.2018.64434

Cañadas, L., Santos-Pastor, M. L. y Castejón, F. J. (2019). Competencias docentes en la formación inicial del profesorado de Educación Física. Retos. 35, 284-288. https://doi.org/10.47197/retos.v0i35.64812

Cardona-Rodríguez, A., Unceta-Satrustegui, K. y Barandiaran-Galdós, M. (2016). Comprehensive pertinence in the quality assessment of higher education. Cultura y Educación, 28(2), 344-358.

https://doi.org/10.1080/11356405.2016.1158446

del Valle, S., de la Vega, R. y Rodríguez, M. (2015). Percepción de las competencias profesionales del docente de educación física en primaria y secundaria. Revista Internacional de Medicina y Ciencias de la Actividad Física y el Deporte, 15(59), 507-526. https://doi.org/10.15366/rimcafd2015.59.007 
Díaz, M. (2009). Percepción de competencia del profesorado de Educación Física e inclusión. Revista Internacional de Medicina y Ciencias de la Actividad Física y el Deporte, 9(35), 322-348.

http://hdl.handle.net/10486/3507

Díaz, M. y Aguado, R. (2012). Percepción de competencia del profesorado universitario de educación física con experiencia sobre la tarea como recurso didáctico. Retos. Nuevas Tendencias en Educación Física, Deporte y Recreación, 22, 16-18. http://hdl.handle.net/10486/662859

Falchikov, N. (2005). Improving assessment through student involvement. Practical solutions for aiding learning in higher and further education. Routledge Falmer.

Gallardo, M. A. (2006). La evaluación de competencias profesionales para la inserción de los Maestros de Educación Física. Revista Electrónica de Investigación Psicoeducativa, 4(3), 469-492.

Garello, M. V. y Rinaudo, M.C. (2013). Autorregulación del aprendizaje, feedback y transferencia de conocimiento. Investigación de diseño con estudiantes universitarios. Revista Electrónica de Investigación Educativa, 15(2), 131-147. https://redie.uabc.mx/redie/article/view/451

González, J. y Wagenaar, R. (2003). Tuning educational structures in Europe. Final Report. Phase One. Universidad de Deusto.

Hamodi, C., López-Pastor, V. M. y López-Pastor, A. T. (2017). If I experience formative assessment whilst studying at university, will I put it into practice later as a teacher? Formative and shared assessment in Initial Teacher Education (ITE). European Journal of Teacher Education, 40(2), 171-190.

https://doi.org/10.1080/02619768.2017.1281909

Hortigüela, D., Palacios, A. y López-Pastor, V. M. (2018). The impact of formative and shared or coassessment on the acquisition of transversal competences in higher education. Assessment \& Evaluation in Higher Education, 44(6), 933-945. https://doi.org/10.1080/02602938.2018.1530341

Kovac, M., Sloan, S. y Starc, G. (2008). Competencies in physical education teaching; Slovenian teachers' views and future perspectives. European Physical Education Review, 3(14), 299-323.

https://doi.org/10.1177/1356336X08095668

López-Pastor, V. M. y Sicilia-Camacho, A. (2017). Formative and shared assessment in higher education. Lessons learned and challenges for the future. Assessment y Evaluation in Higher Education, 42(1), 77-97. https://doi.org/10.1080/02602938.2015.1083535

López-Varas, F. (2015). Relaciones entre competencias, inteligencia y rendimiento académico en alumnos de Grado en Ciencias de la Actividad Física y el Deporte [Tesis doctoral]. Universidad Europea de Madrid. http://hdl.handle.net/11268/4359

Marina, J. A., Pellicer, C. y Manso, J. (2015). Libro blanco de la profesión docente y su entorno escolar. Ministerio de Educación Cultura y Deporte. http://repositorio.minedu.gob.pe/handle/123456789/4621

Martín, R. y de Juanas, Á. (2009). La formación inicial en competencias valorada por los maestros en activo. Revista Electrónica Interuniversitaria de Formación del Profesorado, 12(3), 59-69.

https://redined.mecd.gob.es/xmlui/bitstream/handle/11162/150743/1254435799.pdf?sequence=1\&isAll owed $=y$ 
Martínez-Izaguirre, M., Yániz-Álvarez, C. y Villardón-Gallego, L. (2018). Autoevaluación y reflexión docente para la mejora de la competencia profesional del profesorado en la sociedad del conocimiento. Revista de Educación a Distancia, 56(10), 1-30. https://doi.org/10.6018/red/56/10

Ní Chróinín, D. y O’Sullivan, M. (2014). From initial teacher education through induction and beyond: a longitudinal study of primary teacher beliefs. Irish Educational Studies, 33(4), 451-466.

https://doi.org/10.1080/03323315.2014.984387

Panadero, E., Broadbent, J., Boud, D. y Lodge, J. M. (2018). Using formative assessment to influence selfand co-regulated learning: the role of evaluative judgement. European Journal of Psychology of Education, 34, 535-557. https://doi.org/10.1007/s10212-018-0407-8

Pazo, C. I. y Tejada, J. (2012). Las competencias profesionales en Educación Física. Retos. Nuevas Tendencias en Educación Física, Deporte y Recreación, 22, 5-8.

Perrenoud, P. (2004). Diez nuevas competencias para enseñar. Invitación al viaje. Graó.

Pulido, J. I. (2008). Competencias genéricas ¿Qué son? En Instituto de Ciencias de la Educación, en Competencias genéricas y transversales de los titulados universitarios (pp. 38-45). ICE de la Universidad de Zaragoza. https://ice.unizar.es/sites/ice.unizar.es/files/users/leteo/publicaciones/col. documentos 08.pdf

Rodríguez-Gómez, I., del Valle, S. y de la Vega, R. (2018). Revisión nacional e internacional de las competencias profesionales de los docentes de Educación Física. Retos. Nuevas Tendencias en Educación Física, Deporte y Recreación, 34, 383-388. https://doi.org/10.47197/retos.v0i34.58609

Romero, C. (2009). Definición de módulos y competencias del maestro con mención en Educación Física. Revista Internacional de Medicina y Ciencias del Deporte, 9(34), 179-200.

http://cdeporte.rediris.es/revista/revista34/artcompetencias124.htm

Romero-Martín, M. R., Castejón-Oliva, F. J., López-Pastor, V. M. y Fraile-Aranda, A. (2017). Evaluación formativa, competencias comunicativas y TIC en la formación del profesorado. Comunicar, 52, 73-82. https://doi.org/10.3916/C52-2017-07

Salcines, l., González-Fernández, N., Ramírez-García, A. y Martínez-Mínguez, L. (2018). Validación de la Escala de Autopercepción de Competencias Transversales y Profesionales de Estudiantes de Educación Superior. Profesorado. Revista de Currículum y Formación de Profesorado, 22(3), 31-51.

https://doi.org/10.30827/profesorado.v22i3.7989

Villardón, L. (2006). Evaluación del aprendizaje para promover el desarrollo de competencias. Educatio Siglo XXI, 24, 57-76 https://revistas.um.es/educatio/article/view/153 